\title{
Analysis of the selection of CDK4/6 inhibitors based on experience using palbociclib
}

\author{
MARIKO KIKUCHI, YOKO TANAKA, MITSUO YOKOTA, HIROSHI NISHIMIYA, \\ HIROSHI KATOH, NORIHIKO SENGOKU and YOSHIMASA KOSAKA
}

Department of Breast and Thyroid Surgery, Kitasato University School of Medicine, Sagamihara, Kanagawa 252-0374, Japan

Received June 28, 2019; Accepted October 17, 2019

DOI: $10.3892 /$ br.2019.1248

\begin{abstract}
Palbociclib is a first-in-class potent oral inhibitor of cyclin-dependent kinase (CDK)4/6 that was approved in the USA in 2015 and in Japan in 2017. Next-generation abemaciclib was approved in the USA and Japan in 2018. The use of palbociclib results in a high frequency of bone marrow suppression, whereas abemaciclib induces a low frequency of bone marrow suppression, but a high incidence of diarrhea. However, the most appropriate uses for these CDK4/6 inhibitors remain unclear. In this study, we analyzed the efficacy and side-effects associated with the use of palbociclib at our hospital and examined the suitability of palbociclib or abemaciclib. Among 35 patients who used palbociclib at our hospital from December, 2017 to December, 2018, the mean age was $39-83$ years. The patients receiving treatment with palbociclib with a combination of drugs included 20 patients $(57 \%)$ receiving fulvestrant, 8 patients $(23 \%)$ receiving letrozole, and 7 patients (20\%) receiving fulvestrant + LH-RH (leuprorelin). Fourteen patients $(40 \%)$ had a history of receiving chemotherapy, and 21 patients $(60 \%)$ had no history of receiving chemotherapy. The number of prior treatment regimens was 0-11 (mean, 2.9). The initial dose of palbociclib was $125 \mathrm{mg}$ for 29 patients (83\%) and $100 \mathrm{mg}$ for 6 patients (17\%). Partial response, stable disease and progressive disease were achieved in $6(17 \%), 19$ cases (54\%) and 10 cases (29\%), respectively. Leukocytopenia was observed in 24 cases, neutropenia was observed in 26 cases, anemia was observed in 13 cases, thrombocytopenia was observed in 15 cases, fatigue was observed in 3 cases and itchy skin was observed in 1 case. When the number of neutrophils prior to palbociclib introduction was $<3,000$, neutropenia of grade 3 or higher was observed in all cases following palbociclib introduction. Thus, in order to avoid grade 3 or higher neutropenia and to maintain relative dose intensity, abemaciclib treatment may be considered for
\end{abstract}

Correspondence to: Dr Yoshimasa Kosaka, Department of Breast and Thyroid Surgery, Kitasato University School of Medicine, 1-15-1 Kitasato, Minami-ku, Sagamihara, Kanagawa 252-0374, Japan

E-mail: y-kosaka@med.kitasato-u.ac.jp

Key words: breast cancer, cdk4/6 inhibitors, palbociclib, abemaciclib cases with neutrophils of $<3,000$ prior to the introduction of a CDK4/6 inhibitor.

\section{Introduction}

In recent years, novel drugs for metastatic and recurrent breast cancer have been approved, and treatment options are increasing. However, as there is no clear consensus on which drug to use and in what order, there has been much debate regarding the choice of treatment. Cyclin-dependent kinase (CDK) $4 / 6$ inhibitors selectively inhibit CDK4 and CDK6, arrest cell cycle progression and suppress tumor growth (1). To date, 3 types of CDK4/6 inhibitors have been developed, and in Japan, 2 drugs, palbociclib and abemaciclib, have been approved. Palbociclib is a newly launched molecular-targeted therapeutic agent for use in inoperable or recurrent breast cancer and was the first selective CDK4/6 inhibitor to be introduced worldwide (2-5). In the PALOMA 3 trial, the median progression-free survival (PFS) times were shown to be 9.2 months in the palbociclib combination group and 3.8 months in the placebo group, with significantly better results in the palbociclib combination group [hazard ratio (HR), 0.42; 95\% confidence interval (CI), 0.32-0.56) (6). Its efficacy and safety have been confirmed in Japan, and the drug was approved in December, 2017 in combination with endocrine therapy for hormone receptor-positive human epidermal growth factor receptor 2-negative breast cancer $(7,8)$. The efficacy and safety of abemaciclib were subsequently validated, and abemaciclib was then approved in Japan in 2018 (9-11). In the MONARCH 2 trial, the median PFS times were 16.4 months for the abemaciclib combination group and 9.3 months for the placebo group, with significantly better results in the abemaciclib combination group (HR, 0.553; 95\% CI, 0.449-0.681) (10). The side-effects associated with the use of CDK4/6 inhibitors are primarily blood toxicity and digestive complications, such as nausea, vomiting and diarrhea. However, the side-effect profiles are somewhat different; palbociclib has been reported to induce a high frequency of hematologic toxicity, and abemaciclib has been reported to induce a high frequency of diarrhea (2-6,9-11). Thus, the appropriate applications for the use of these 2 CDK4/6 inhibitors have not yet been determined.

In this study, we analyzed the efficacy and side-effects associated with the use of palbociclib in patients at our hospital and examined the suitability of palbociclib or abemaciclib based on adverse events. 


\section{Patients and methods}

Patients. This study included 35 patients who were able to continuously receive 2 or more courses of palbociclib from December, 2017 to December, 2018 at Kitasato University Hospital. The patient information is presented in Table I. All patients had recurrent or metastatic hormone-positive breast cancer (stage IV). The ages of the patients ranged from 39 to 83 years (median, 55 years). None of the patients discontinued treatment due to adverse events.

Analysis methods. The effects of palbociclib were evaluated using the response evaluation criteria in solid tumors (RECIST) RECIST. Adverse events were evaluated using CTCAE ver4.1. Factors related to neutropenia of grade 3 or higher were also analyzed.

Statistical analysis. We calculated the average of the disease-free period and PFS. PFS was estimated using the Kaplan-Meier method and P-values were generated using the Wilcoxon test. For categorical variables, $\mathrm{P}$-values were calculated using the Chi-square test. A value of $\mathrm{P}<0.05$ was considered to indicate a statistically significant difference. All statistical analyzes were performed using JMP Pro version 11 software (SAS Institute).

\section{Results}

Patient demographics and clinical data. The patient information is presented in Table I. All patients had stage IV, invasive ductal carcinoma. The ages of the patients ranged from 39 to 83 years (median, 55 years). The patients receiving treatment with a combination of drugs included 20 patients (57\%) with fulvestrant, 8 patients $(20 \%)$ with letrozole, and 7 patients (23\%) with fulvestrant + LH-RH (leuprorelin). Fourteen patients $(40 \%)$ had a history of chemotherapy, and $21(60 \%)$ had no history of chemotherapy. The number of prior treatment regimens (including chemotherapy and hormone therapy) was 0-11 (mean, 2.9). The initial dose of palbociclib was $125 \mathrm{mg}$ for 29 patients $(83 \%)$ and $100 \mathrm{mg}$ for 6 patients (17\%). Patients who were reduced to $100 \mathrm{mg}$ were patients 74 years of age or older, or patients with brain metastases and a performance status (PS) of $\leq 2$.

Analysis of efficacy. The efficacy of the treatment is presented in Table II. There were 6 cases (17\%) of partial response (PR), 19 cases (54\%) of stable disease (SD) and 10 cases (29\%) of progressive disease (PD). The disease-free period averaged 5.5 months (1.17-10.73 months)

Analysis of side-effects. The side-effects of treatment are presented in Table III. There were 24 cases with leukocytopenia (7 cases with grade 3 or higher), 26 cases with neutropenia (16 cases with grade 3 or higher), 13 cases with anemia (1 case with grade 3 or higher) and 15 cases with thrombocytopenia (grade 3 or higher). There were 3 cases of fatigue, 1 case of itchy skin and 1 case of febrile neutropenia $(\mathrm{FN})$.

Riskfactors for severe neutropenia. Subsequently, we examined the expression of neutropenia of grade 3 or higher (Table IV). There were no significant differences in the presence or absence of prior chemotherapy treatment, the number of regimens, or
Table I. Patient background.

\begin{tabular}{|c|c|}
\hline Parameter & Numbe \\
\hline \multicolumn{2}{|l|}{ Age (years) } \\
\hline \multicolumn{2}{|l|}{ Combination drugs } \\
\hline Fulvestrant & 20 \\
\hline Fulvestrant + LH-RH & 7 \\
\hline Letrozole & 8 \\
\hline \multicolumn{2}{|l|}{ Initial dose } \\
\hline $125 \mathrm{mg}$ & 29 \\
\hline $100 \mathrm{mg}$ & 6 \\
\hline \multicolumn{2}{|l|}{ Prior treatment regimens } \\
\hline Chemotherapy only & 6 \\
\hline Hormone therapy only & 13 \\
\hline Both chemotherapy and hormone therapy & 8 \\
\hline No prior treatment history & 8 \\
\hline \multicolumn{2}{|l|}{ Number of prior treatment regimens } \\
\hline 0 & 8 \\
\hline 1 & 3 \\
\hline 2 & 7 \\
\hline 3 & 10 \\
\hline More than 4 regimens & 7 \\
\hline \multicolumn{2}{|l|}{ Metastatic site } \\
\hline Lymph node & 20 \\
\hline Bone & 20 \\
\hline Lung & 16 \\
\hline Liver & 13 \\
\hline Brain & 2 \\
\hline Pleural dissemination & 4 \\
\hline
\end{tabular}

age. Although the presence or absence of bone metastasis did not differ significantly, bone metastasis cases tended to exhibit neutropenia of grade 3 or higher $(\mathrm{P}=0.09)$. In patients with $<3,000$ neutrophils prior to palbociclib induction, neutropenia of grade 3 or higher was observed in all 12 patients $(\mathrm{P}<0.0001)$.

Dose reduction. The dose of pabociclib was reduced in 13 patients (37\%). Of these, 7 cases had 1 dose reduction, and 6 cases had a 2 -stage dose reduction. PFS was improved in patients who did not have a dose reduction $(\mathrm{P}=0.04$, Wilcoxon test). The median PFS (95\% CI) was 6.3 months $(5.6-\mathrm{NE})$ in patients who did not have a dose reduction, and the median PFS (95\% CI) was 3.3 months (2.8-NE) in patients who had a level 1 or 2 dose reduction (Fig. 1).

\section{Discussion}

Currently, there are no clear guidelines regarding the use of palbociclib and abemaciclib. In this study, in cases in which the number of neutrophils prior to the introduction of a CDK4/6 inhibitor was $<3,000$ or less, neutropenia of grade 3 or higher was observed in all cases owing to palbociclib. We believe that abemaciclib may be selected in cases in which the number 
Table II. Efficacy of palbociclib treatment $(n=35)$.

\begin{tabular}{lcc}
\hline Response & Number & $\%$ \\
\hline Partial response $^{\mathrm{a}}$ & 6 & 17 \\
Stable disease $^{\mathrm{b}}$ & 19 & 54 \\
Progressive disease $^{\mathrm{c}}$ & 10 & 29
\end{tabular}

apartial response: Compared to the baseline diameter, the diameter of

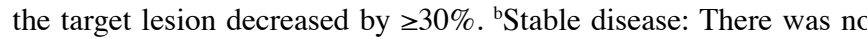
reduction equivalent to partial response and no increase equivalent to progressive disease compared to the smallest diameter sum in

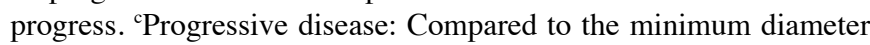
in progress, the diameter of the target lesion increased by $\geq 20 \%$, and the absolute diameter increased by $\geq 5 \mathrm{~mm}$ in absolute value.

Table III. Side-effects associated with palbociclib treatment.

\begin{tabular}{lcccccc}
\hline & \multicolumn{2}{c}{ All grades } & & \multicolumn{2}{c}{$\begin{array}{c}\text { Higher than } \\
\text { grade } 3\end{array}$} \\
\cline { 2 - 3 } \cline { 6 - 7 } Side-effect & Number & $\%$ & & Number & $\%$ \\
\hline Leukocytopenia & 24 & 69 & & 7 & 20 \\
Neutropenia & 26 & 74 & & 16 & 46 \\
Anemia & 13 & 37 & & 1 & 3 \\
Thrombocytopenia & 15 & 43 & & 0 & 0 \\
Fatigue & 3 & 9 & & 0 & 0 \\
Itchy skin & 1 & 3 & & 0 & 0 \\
Febrile neutropenia & 1 & 3 & & 1 & 3 \\
\hline
\end{tabular}

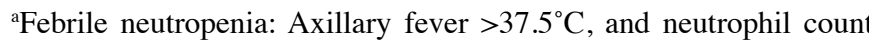
$<500 / \mu 1$, or expected to decrease to $<500 / \mu 1$ within $48 \mathrm{~h}$.

of neutrophils prior to introduction of the CDK4/6 inhibitor is $<3,000$.

CDK4/6 inhibitors are agents that block the cell cycle and lead to similar side-effects as cytotoxic preparations (1). The use of palbociclib frequently leads to side-effects associated with the blood system. In the PALOMA-3 trial, neutropenia, anemia and thrombocytopenia were detected in 79, 26 and $19 \%$ of cases when palbociclib was used in combination with fulvestrant (6); in particular, neutropenia of grade 3 or higher was as high as $62 \%$, and the incidence of adverse events of febrile neutopenia was $0.9 \%$. Although not frequent, we experienced 1 case of febrile neutropenia, in which hospitalization was required; thus, the occurrence of febrile neutopenia should be avoided. Moreover, in the PALOMA-3 trial, the proportion of patients experiencing withdrawal of palbociclib for neutropenia was $59.7 \%$, and the proportion of patients experiencing dose reduction was $27 \%$. By contrast, in the MONARCH 2 test, when administered a combination of fulvestrant and abemaciclib, the incidences of neutropenia, anemia, thrombocytopenia and neutropenia of grade 3 or higher were 46, 29, 15 and $26 \%$, respectively (10).

The side-effect of cytopenia was lower for abemaciclib compared with that of palbociclib. Palbociclib has not been
Table IV. Risk factors for severe neutropenia.

\begin{tabular}{|c|c|c|c|}
\hline \multirow[b]{2}{*}{ Risk factor } & \multicolumn{2}{|c|}{$\begin{array}{l}\text { Number of patients } \\
\text { with neutropenia of } \\
\text { grade } 3 \text { or higher }(\%)\end{array}$} & \multirow[b]{2}{*}{ P-value } \\
\hline & - & + & \\
\hline \multicolumn{4}{|c|}{ Chemotherapy history } \\
\hline+ & $8(57)$ & $6(43)$ & $\mathrm{P}=0.09$ \\
\hline- & $6(29)$ & $15(71)$ & \\
\hline \multicolumn{4}{|c|}{ The number of regimens } \\
\hline$<4$ & $12(43)$ & $16(57)$ & $\mathrm{P}=0.48$ \\
\hline$\geq 4$ & $2(29)$ & $5(71)$ & \\
\hline \multicolumn{4}{|l|}{ Age (years) } \\
\hline$<50$ & $3(33)$ & $6(67)$ & $\mathrm{P}=0.63$ \\
\hline$\geq 50$ & $11(42)$ & $15(58)$ & \\
\hline \multicolumn{4}{|c|}{ Bone metastasis } \\
\hline- & $8(57)$ & $6(43)$ & $\mathrm{P}=0.09$ \\
\hline+ & $6(29)$ & $15(71)$ & \\
\hline \multicolumn{4}{|c|}{$\begin{array}{l}\text { Number of neutrophils } \\
\text { prior to palbociclib induction }\end{array}$} \\
\hline$<3,000$ & $0(0)$ & $12(100)$ & $\mathrm{P}<0.0001$ \\
\hline$\geq 3,000$ & $14(61)$ & $9(39)$ & \\
\hline
\end{tabular}

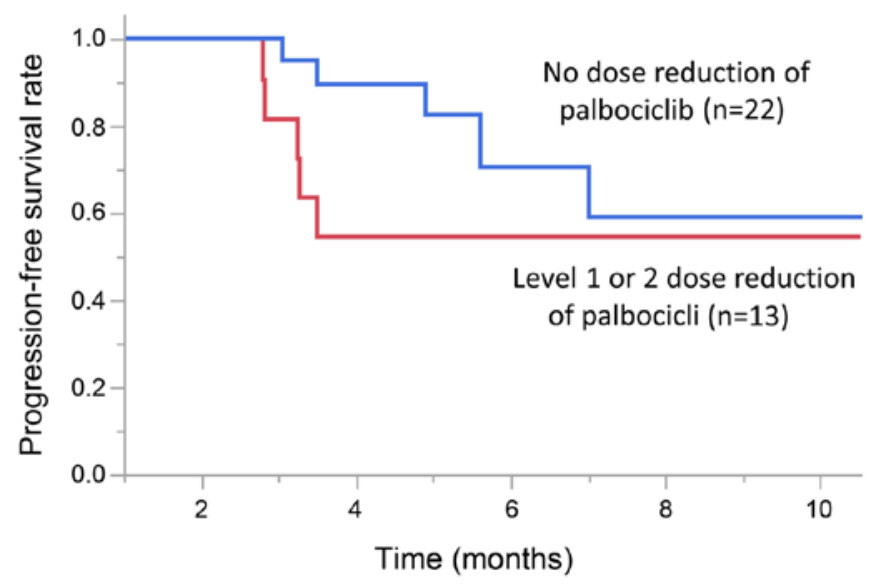

Figure 1. Progression-free survival in patients with or without dose reduction. Progression-free survival was improved in patients who did not have a dose reduction ( $\mathrm{P}=0.04$, Wilcoxon test). The median PFS $(95 \% \mathrm{CI})$ was $6.3 \mathrm{M}$ (5.6-NE) in patients who had no dose reduction, and the median PFS (95\% CI) was $3.3 \mathrm{M}(2.8-\mathrm{NE})$ in patients who had a level 1 or 2 dose reduction.

reported to result in differences in PFS between those who had dose reductions of 1 or more levels $(n=100)$ and those who did not have dose reductions $(n=245)(12)$. However, no analysis has been conducted for patients who had dose reductions in 1 or 2 stages. In the current study, the initial dose of palbociclib was $125 \mathrm{mg}$ for 29 patients $(83 \%)$ and $100 \mathrm{mg}$ for 6 patients (17\%). Patients who were reduced to $100 \mathrm{mg}$ were patients 74 years of age or older, or patients with brain metastases and PS2 or greater. PFS was significantly improved in patients who did not have a dose reduction. In addition, there were 6 cases in which a 2-stage dose reduction was required, 
indicating a relatively high frequency. The current study differs from previous clinical trials. However, blood levels [maximum blood concentration, Cmax and the area under the blood concentration-time curve (AUC)] are proportional to the dose (13). When the relative dose intensity (RDI) decreases, the clinical effect may not be sufficient. However, since the sample size was small in this study, verification with a larger sample size is also necessary. Thus, in order to maintain the RDI, it is better to avoid drug withdrawal and dose reduction as much as possible in most cases. In order to avoid the appearance of neutropenia of grade 3 or higher and to maintain RDI, it is suggested that abemaciclib should be considered for cases with neutrophils of $<3,000$ prior to the introduction of a CDK4/6 inhibitor.

In conclusion, the results revealed that palbociclib administration was extremely likely to cause neutropenia of grade 3 or higher in cases in which the number of neutrophils was $<3,000$ prior to induction. Therefore, such cases should be treated with neutropenia in mind.

\section{Acknowledgements}

Not applicable.

\section{Funding}

No funding was received.

\section{Availability of data and materials}

All data generated or analyzed during this study are included in this published article or are available from the corresponding author on reasonable request.

\section{Authors' contributions}

YT, MY, HN, HK and NS designed the study, and wrote the initial draft of the manuscript. YK contributed to the analysis and interpretation of the data, and assisted in the preparation of the manuscript. All authors (MK, YT, MY, HN, HK, NS and YK) have contributed to data collection and interpretation, and have critically reviewed the manuscript. All authors have read and approved the final version of the manuscript, and agree to be accountable for all aspects of the work in ensuring that questions related to the accuracy or integrity of any part of the work are appropriately investigated and resolved.

\section{Ethics approval and consent to participate}

This study is a retrospective study. This study was approved by the Kitasato University Hospital Ethics Committee (B18-294). Our survey began on May 17, 2019.

\section{Patient consent for publication}

Not applicable.

\section{Competing interests}

The authors declare that they have no competing interests.

\section{References}

1. Fry DW, Harvey PJ, Keller PR, Elliott WL, Meade M, Trachet E, Albassam M, Zheng X, Leopold WR, Pryer NK, et al: Specific inhibition of cyclin-dependent kinase 4/6 by PD 0332991 and associated antitumor activity in human tumor xenografts. Mol Cancer Ther 3: 1427-1438, 2004.

2. European Medicines Agency: IBRANCE Product Information. http://www.ema.europa.eu/en/medicines/human/EPAR/ibrance.

3. Pharmaceuticals and Medical Devices Agency: New drugs approved in September (2017) (In Chinese). https://www.pmda. go.jp/index.html.

4. Ibrance (palbociclib): Full prescribing information. Pfizer Inc., New York, 2018.http://labeling.pfizer.com/ShowLabeling. aspx?id=2191.

5. Finn RS, Martin M, Rugo HS, Jones S, Im SA, Gelmon K, Harbeck N, Lipatov ON, Walshe JM, Moulder S, et al: Palbociclib and letrozole in advanced breast cancer. N Engl J Med 375: 1925-1936, 2016.

6. Turner NC, Ro J, André F, Loi S, Verma S, Iwata H, Harbeck N, Loibl S, Huang Bartlett C, Zhang K, et al; PALOMA3 Study Group: Palbociclib in hormone receptor positive advanced breast cancer. N Engl J Med 373: 209-219, 2015.

7. Tamura K, Mukai H, Naito Y, Yonemori K, Kodaira M, Tanabe Y, Yamamoto N, Osera S, Sasaki M, Mori Y, et al: Phase I study of palbociclib, a cyclin-dependent kinase 4/6 inhibitor, in Japanese patients. Cancer Sci 107: 755-763, 2016.

8. Masuda N, Nishimura R, Takahashi M, Inoue K, Ohno S, Iwata H, Mori Y,Hashigaki S, Muramatsu Y, Nagasawa T, et al: Palbociclib in combination with letrozole as first-line treatment for advanced breast cancer: A Japanese phase II study. Cancer Sci 109: 803-813, 2018.

9. Dickler MN, Tolaney SM, Rugo HS, Cortés J, Diéras V, Patt D, Wildiers H, Hudis CA, O'Shaughnessy J, Zamora E, et al: MONARCH 1, a phase II study of abemaciclib, a CDK4 and CDK6 inhibitor, as a single agent, in patients with refractory $\mathrm{HR}^{+} / \mathrm{HER} 2-$ metastatic breast cancer. Clin Cancer Res 23: 5218-5224, 2017.

10. Sledge GW Jr, Toi M, Neven P, Sohn J, Inoue K, Pivot X, Burdaeva O, Okera M, Masuda N, Kaufman PA, et al: MONARCH 2: Abemaciclib in combination with fulvestrant in women with $\mathrm{HR}^{+} / \mathrm{HER} 2-$ advanced breast cancer who had progressed while receiving endocrine therapy. J Clin Oncol 35: 2875-2884, 2017.

11. Goetz MP, Toi M, Campone M, Sohn J, Paluch-Shimon S, Huober J, Park IH, Trédan O, Chen SC, Manso L, et al: MONARCH 3: Abemaciclib as initial therapy for advanced breast cancer. J Clin Oncol 35: 3638-3646, 2017.

12. Loibl S, Turner NC, Ro J, Cristofanilli M, Iwata H, Im SA, Masuda N, Loi S, André F, Harbeck N, et al: Palbociclib combined with fulvestrant in premenopausal women with advanced breast cancer and prior progression on endocrine therapy: PALOMA-3 results. Oncologist 22: 1028-1038, 2017.

13. Pfizer Internal Materials: Pharmacokinetics in Japanese healthy adults (single administration) [L20170116042]. https://www. pfizer.com/science/find-a-trial/search/Ibrance.

This work is licensed under a Creative Commons Attribution-NonCommercial 4.0 International (CC BY-NC 4.0) License. 